\title{
Effect of preoperative anxiety on spectral entropy during induction with propofol
}

\author{
Yun Hong Kim and Won-Jun Choi \\ Department of Anesthesiology and Pain Medicine, Sungkyunkwan University School of Medicine, Kangbuk Samsung Hospital, \\ Seoul, Korea
}

Background: Anxiety influences brain wave activity. E-Entropy module-derived spectral entropy is an electroencephalographic derivative used to monitor the depth of sedation. This study assessed the effect of preoperative anxiety on the spectral entropy parameters of response entrophy (RE) and state entrophy (SE).

Methods: Trait anxiety was measured in 92 American Society of Anesthesiologists physical status I-II patients with the Spielberger State-Trait Anxiety Inventory (STAI) form X2 and state anxiety with STAI-X1 just before anesthesia. RE, $\mathrm{SE}$, blood pressure and heart rate were measured before induction. Propofol was infused via a target controlled infusion pump. At loss of consciousness (LOC), the effect-site concentration (Ce), RE, SE and total amount of propofol were recorded. Patients were stratified into three groups based on their state and trait anxiety scores to evaluate the effect of anxiety level on entropy values.

Results: STAI-X1 was significantly correlated with RE and SE for LOC $(\rho=0.230, \mathrm{P}=0.028$ and $\rho=0.308, \mathrm{P}=0.003$, respectively) and also with STAI-X2 $(\rho=0.411, \mathrm{P}=0.001$, respectively). SE was higher in the high state anxiety group than in the low state anxiety group $(\mathrm{P}=0.017)$. The other measured variables were not correlated with any anxiety scales. Conclusions: The state of anxiety increases RE and SE values at LOC induced with propofol. High state anxiety is associated with higher SE than apparent at low state anxiety. When determining the propofol-induced LOC by spectral entropy, anxiety levels should be considered. (Korean J Anesthesiol 2013; 65: 108-113)

Key Words: Anxiety, Electroencephalography, Entropy, Propofol.

Received: September 28, 2012. Revised: 1st, December 28, 2012; 2nd, January 5, 2013. Accepted: January 20, 2013.

Corresponding author: Won-Jun Choi, M.D., Department of Anesthesiology and Pain Medicine, Sungkyunkwan University School of Medicine, Kangbuk Samsung Hospital, 108, Pyeong-dong, Jongno-gu, Seoul 110-746, Korea. Tel: 82-2-2001-2315, Fax: 82-2-2001-2326, E-mail: wj2006. choi@samsung.com

(c) This is an open-access article distributed under the terms of the Creative Commons Attribution Non-Commercial License (http:// creativecommons.org/licenses/by-nc/3.0/), which permits unrestricted non-commercial use, distribution, and reproduction in any medium, provided the original work is properly cited. 


\section{Introduction}

E-Entropy module-derived spectral entropy is an electroencephalographic derivative used to monitor the depth of sedation during general anesthesia. The entropy values of response entropy (RE) and state entropy (SE) are available indices to detect loss of consciousness (LOC) induced with propofol [1,2]. Brain wave activities measured by electroencephalography are determined by various psychopathological conditions [3]. Even in healthy adults, anxiety status induces changes of brain wave patterns [4-6]. Therefore, spectral entropy values may be altered by the degree of anxiety.

We investigated the effect of preoperative anxiety on the spectral entropy parameters and investigated whether levels of trait or preoperative state anxiety affected the E-Entropy modulederived spectral entropy values before propofol induction of LOC and at the time of LOC.

\section{Materials and Methods}

Ethical approval for this study performed between August 2010 and January 2012 was provided by the relevant Institutional Review Board. Ninety two patients were enrolled in this prospective study. Written informed consent was obtained from each patient. Inclusion criteria were female gender, normal thyroid function test, age between 18-65 years, American Society of Anesthesiologists I and II status and elective thyroidectomy for thyroid cancer including both open and laparoscopic approaches, which is classified as minor surgery [7]. Exclusion criteria were history of previous general anesthesia experiences or psychiatric disorders, drug dependence and/or alcohol abuse, use of anxiolytic medication, smoking history for the significant correlation between smoking history and higher anxiety [7] and inability to attend consultation and hearing.

The Korean version of the Spielberger State-Trait Anxiety Inventory (STAI) form X was adopted for measuring preoperative anxiety [8]. STAI-X is subdivided into two different scales, STAI-X1 and -X2, used to evaluate state anxiety and trait anxiety, respectively. Each scale is comprised of 20 selfevaluation questions, recommended to be filled out by the patients through self-assessment. One day before surgery, patients completed the STAI-X2 questionnaire to measure the degrees of innate anxiety. Premedication was not administered to any patient. Immediately after the admission into the surgery waiting room, the patients were asked to fill out the STAI-X1 questionnaire, which reflects the current anxiety level before anesthetic induction. When the patients lay down in the surgery room, entropy monitor sensor electrodes of an E-Entropy module (GE Healthcare Finland Oy, Helsinki, Finland) were placed above the eyebrows as recommended by the manufacturer. Anesthesia was induced by infusing 2\% propofol (Fresofol MCT 2\% Inj.; Fresenius Kabi Austria $\mathrm{GmbH}$, Graz, Austria) using a Marsh model of commercial target controlled infusion (TCI) pump (Orchestra ${ }^{\circledR}$ Base Primea, Fresenius Vial, Brezins, France) at an initial setting of the effect-site concentration (Ce) of $0.5 \mu \mathrm{g} / \mathrm{ml}$. Subsequently, the target Ce was adjusted in 30-second increments of $0.5,0.3$ and finally $0.1 \mu \mathrm{g} / \mathrm{ml}$ as the score of the Observer Assessment of Alertness/Sedation (OAA/S) scale reached 4, 3 and 2, respectively (Table 1) [9], similar to a previously described method [10]. The Ce of propofol, and SE and RE at LOC were measured by an assigned anesthesiologist who was blinded to the anxiety level of the patient anxiety. Ce at the point of LOC was designated corresponding to the OAA/S scale $<2$, similar to lthe loss of response both to verbal commands and mild shaking in the shoulder (Table 1) [9]. The total amount of propofol required for LOC and the time from induction to LOC were recorded. During the measurement of variables, the patients were supplied with $100 \% \mathrm{O}_{2}$ by a facial mask without stimulation, and the presence of spontaneous breathing was monitored. After data collection, the scheduled surgery was conducted under general anesthesia. The data of hemodynamic variables including heart rate (HR), and systolic and diastolic blood pressure (BP) were determined as the mean of three consecutive measurements just before the induction.

Statistical analyses were performed by using the PASW statistics 18.0 Statistical Software Package (SPSS Inc, Chicago, IL, USA). According to a previous study [6], a Spearman correlation coefficient $(\rho)$ between STAI-trait anxiety scores and $\mathrm{SE}$ before the induction was assumed to be 0.3 . Based on the analysis with $\operatorname{IBM}^{\circledR}$ SPSS $^{\circledR}$ SamplePower ${ }^{\circledR}$ 3.0.1), the sample size of 81 subjects was calculated to obtain a power of $80 \%$ and an $\alpha$ of 0.05 for the Spearman correlation test. Thus, 92 patients were selected for this prospective study.

To determine the variables correlated to anxiety variables, STAI-X1 and -X2 were respectively compared to RE and SE before the induction and at LOC, Ce at LOC, total propofol requirement to LOC, time from induction to LOC and hemodynamic variables before the anesthetic induction (systolic and diastolic BP, and HR) by the Spearman correlation test. Also,

Table 1. The Observer Assessment of Alertness/Sedation (OAA/S) Scale [9]*

\begin{tabular}{lc}
\hline \multicolumn{1}{c}{ Content } & Score \\
\hline Responds readily to name spoken in normal tone & 5 \\
Responds lethargically to name spoken in normal tone & 4 \\
Responds only after name is called loudly, repeatedly, or both & 3 \\
Responds only after mild prodding or shaking & 2 \\
Responds only after painful trapezius squeeze & 1 \\
Does not respond to painful trapezius squeeze & 0
\end{tabular}

*J Clin Psychopharmacol 1990; 10: 244-51. 
to evaluate the effect of anxiety level on entropy values, the Kluskal-Wallis test was applied after the cohort of patients were stratified into three groups based on their state and trait anxiety scores: low-anxiety group (<25\% STAI scores), medium-anxiety group (25-75\% STAI scores), and high-anxiety group (> 75\% STAI scores). The Mann-Whitney U test was used for posthoc comparisons with Bonferroni correction. The result was statistically significant with a $\mathrm{P}$ value $<0.05$.

Table 2. Patient Characteristics

\begin{tabular}{lc}
\hline Number of patients & 92 \\
Age $(\mathrm{yr})$ & $41.7 \pm 9.3$ \\
Weight $(\mathrm{kg})$ & $58.1 \pm 8.1$ \\
Height $(\mathrm{cm})$ & $158.9 \pm 5.5$ \\
Body mass index $\left(\mathrm{kg} / \mathrm{m}^{2}\right)$ & $23.1 \pm 3.4$ \\
Open thyroidectomy/laparoscopic thyroidectomy & $58 / 34$ \\
\hline
\end{tabular}

Values are mean \pm SD or number.

Table 3. Data Profile of Variables for Spearmann Correlation Test

\begin{tabular}{lc}
\hline STAI-X2 before surgery & $46(42-50)$ \\
STAI-X1 before induction & $41(28-46)$ \\
Ce of propofol at loss of consciousness & $3.5(3.0-3.8)$ \\
Systolic blood pressure before induction $(\mathrm{mmHg})$ & $127.7 \pm 10.0$ \\
Diastolic blood pressure before induction $(\mathrm{mmHg})$ & $68.9 \pm 12.7$ \\
Heart rate before induction (beats/min) & $68.9 \pm 12.3$ \\
Response entropy before induction & $97(96-98)$ \\
Response entropy at LOC & $59(51-68)$ \\
State entropy before induction & $88(86-90)$ \\
State entropy at LOC & $55(48-62)$ \\
\hline
\end{tabular}

Number of patients $=92$. Values are presented as median (interquartile range) or mean \pm SD. STAI: the Spielberger State-Trait Anxiety Inventory, STAI-X1: STAI scale for state anxiety, STAI-X2: STAI scale for trait anxiety, Ce: effect-site concentration, LOC: loss of consciousness.

\section{Results}

The characteristics of the enrolled patients and the data profile for the Spearman correlation test are presented in Table 2 and 3. There were no missing subject data.

The preoperative STAI-X1 score was positively correlated with RE and SE at LOC ( $\rho=0.230$ with $\mathrm{P}=0.028$ and $\rho=$ 0.308 with $\mathrm{P}=0.003$, respectively) (Fig. 1 , Table 4 ). $\mathrm{RE}$ and SE values before anesthetic induction were not influenced by preanesthetic STAI-X1 levels. No correlation was evident between STAI-X2 and entropy values at any measured point although the STAI-X2 score was associated with the preoperative STAI-X1 score in a positive manner $(\rho=0.411, \mathrm{P}=0.001)$ (Table 4$)$. Systolic and diastolic BP and heart rate before the induction were not correlated to anxiety levels. Also, the Ce of propofol for LOC was not affected by any anxiety scale. Stratification into three groups based on anxiety did not reveal any demographic differences. When comparing the entropy values among low, medium and high anxiety groups, no influence of STAI-X2 was apparent on the degree of RE and SE before induction, and at LOC there were no differences in total propofol requirement

Table 4. Relationship between Two Variables with Significant Spearmann Correlation Coefficient $(\rho)$

\begin{tabular}{lcc}
\hline \multicolumn{1}{c}{ Variable 1 vs Variable 2 } & Spearmann's rho $(\rho)$ & P value \\
\hline STAI-X1 vs STAI-X2 & 0.411 & 0.001 \\
STAI-X1 vs Respones entropy at LOC & 0.230 & 0.028 \\
STAI-X1 vs State entropy at LOC & 0.308 & 0.003 \\
\hline
\end{tabular}

STAI: Spielberger State-Trait Anxiety Inventory, STAI-X1: STAI scale for state anxiety, STAI-X2: STAI scale for trait anxiety, LOC: loss of consciousness.
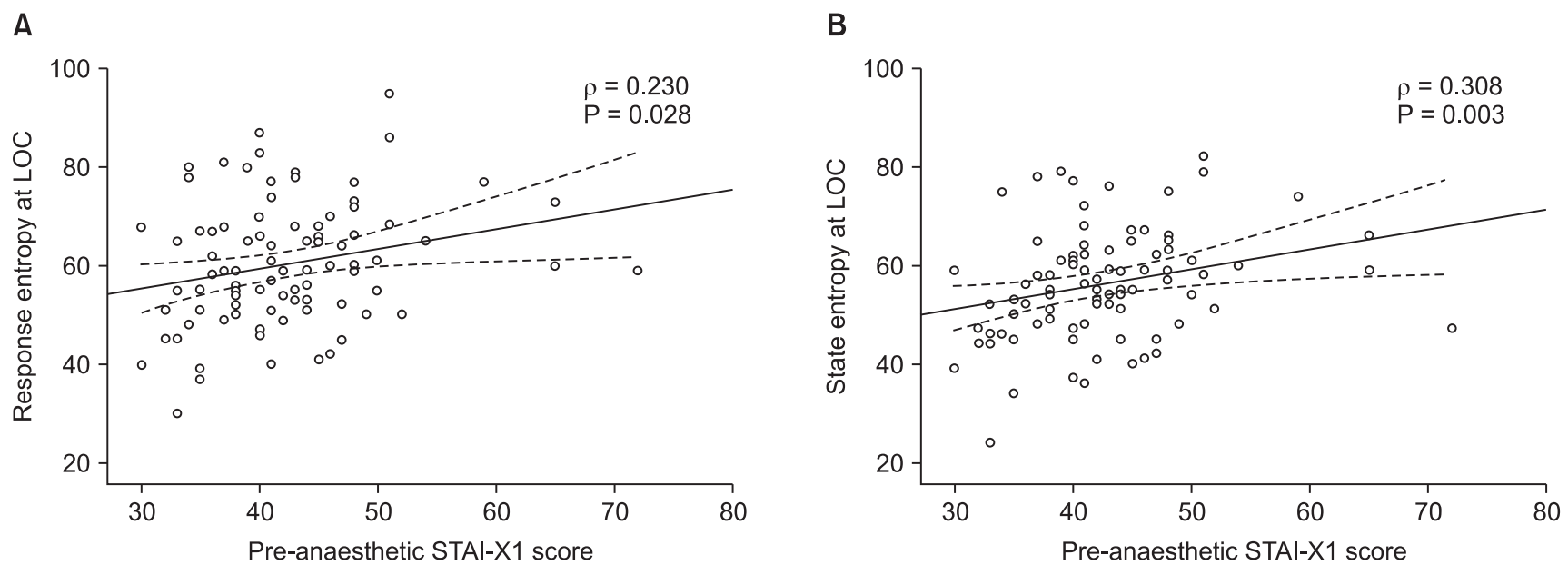

Fig. 1. The correlation between the Spielberger State-Trati Anxiety Inventory (STAI) form X1 score during pre-anesthetic period and Response entropy (RE) and State entropy (SE), at loss of consciousness (LOC) under propofol induction. STAI-X1 was significantly correlated with RE and SE for $\operatorname{LOC}([\mathrm{A}] \rho=0.230, \mathrm{P}=0.028$ and $[\mathrm{B}] \rho=0.308, \mathrm{P}=0.003$, respectively). 
Table 5. Comparisons between Different Trait Anxiety Score Groups with the RE and SE during Induction and LOC, Concentration of the Effective Site and Total Propofol Requirement to LOC

\begin{tabular}{|c|c|c|c|c|}
\hline \multicolumn{5}{|c|}{ STAI-X2 } \\
\hline & Low group & Middle group & High group & $P$ value \\
\hline $\mathrm{N}$ & 21 & 47 & 24 & \\
\hline Response entropy induction & $98(97-98)$ & $97(96-98)$ & $97.5(96.0-98.8)$ & 0.275 \\
\hline State entropy induction & $89(87.5-90.0)$ & $88(85-90)$ & $87.5(83.3-89.8)$ & 0.476 \\
\hline RE LOC & $61(54.0-75.5)$ & $56(50-66)$ & $60.5(52.3-68.0)$ & 0.374 \\
\hline SE LOC & $52(47.5-70.0)$ & $54(46-61)$ & $58.5(52.8-64.8)$ & 0.359 \\
\hline $\mathrm{Ce}$ & $3.4(2.65-3.55)$ & $3.4(3.0-3.8)$ & $3.7(3.0-3.9)$ & 0.094 \\
\hline Propofol requirement (mg) & $103(83-115)$ & $103(88.5-113.3)$ & $107.5(89.8-116.0)$ & 0.671 \\
\hline
\end{tabular}

Data are number or median (interquartile range). Stratified into three groups based on their anxiety scores: Low group $(<25 \%$ STAI scores), Middle group (25\%-75\% STAI scores), High group (> 75\% STAI score). STAI: Spielberger State-Trait Anxiety Inventory, STAI-X2: STAI scale for trait anxiety, RE: Response Entropy, SE: State Entropy, Ce: effect-site concentration, LOC: loss of consciousness.

Table 6. Comparisons between Different State Anxiety Score Groups with the RE and SE during Induction and LOC, and Concentration of Effective Site

\begin{tabular}{lcccc}
\hline & & & & \\
& STAI-X1 & High group & \\
\hline $\mathrm{N}$ & Low group & Middle group & 25 & P value \\
Response entropy induction & 23 & 44 & $97(95.5-98.0)$ & 0.386 \\
State entropy induction & $89(97-98)$ & $97(97-99)$ & $87(83.0-89.5)$ & 0.274 \\
RE LOC & $55(45-60)$ & $58(87-90)$ & $51(57.0-72.5)$ & 0.159 \\
SE LOC & $50(44-58)$ & $55(49.5-62.0)$ & $59(52.5-66.0)^{*}$ & 0.017 \\
Ce & $3.4(3.0-3.7)$ & $3.5(3.0-3.9)$ & $3.0(2.9-3.7)$ & 0.199 \\
Propofol requirement (mg) & $102(90-115)$ & $103(89.0-113.3)$ & $106.2(87.6-116.0)$ & 0.906 \\
\hline
\end{tabular}

Data are number or median (interquartile range). Stratified into three groups based on their anxiety scores: Low group (<25\% STAI scores), Middle group (25\%-75\% STAI scores), High group (> 75\% STAI score). STAI: Spielberger State-Trait Anxiety Inventory, STAI-X1: STAI scale for state anxiety, RE: Response Entropy, SE: State Entropy, Ce: effect-site concentration, LOC: loss of consciousness. $* \mathrm{P}<0.05$ when compared with low group under Bonferroni correction (adjusted $\mathrm{P}=0.027$ ).

to LOC (Table 5). However, pre-anesthetic STAI-X1 revealed a statistically significant difference in SE at LOC although there was no discrepancies in RE and SE before induction and RE at LOC. Especially, the STAI-X1 high group ( $>46$ ) at LOC presented significantly higher levels of SE than the STAI-X1 low group $(<37.25)(\mathrm{P}=0.017)$. There were no differences in total propofol requirement to LOC (Table 6).

\section{Discussion}

Our study revealed a lack of correlation of trait and preanesthetic state anxieties with both pre-anesthetic RE and SE. State anxiety levels during pre-anesthetic periods exerted a strong influence on the values of SE under propofol-induced LOC. Based on correlation analysis, SE and RE values at LOC were significantly associated with the degree of state anxiety. However, the correlation between RE and pre-anesthetic state anxiety scores was weaker than that between SE and state anxiety scores, even though both correlations were statistically significant (Table 4). After the patients were categorized into three groups based on the interquartile range for state anxiety scores [11,12], RE values at LOC did not show a significant difference among preoperative state anxiety groups. On the other hand, SE values at LOC were significantly different, especially between low and high anxiety, during pre-anesthetic periods. Presently, RE and SE values at LOC did not have the same effectiveness in discriminating the levels of anxiety. The exact mechanism for such differences in the influence of anxiety between RE and SE remains unclear. It is conceivable that RE covers the electromyographic (EMG) components.

Electroencephalography (EEG) waveforms are generally classified according to their frequency: alpha $(7.5-12.5 \mathrm{~Hz})$, beta $(12.5-35 \mathrm{~Hz})$, theta $(3.5-7.5 \mathrm{~Hz})$, delta $(1.5-3.5 \mathrm{~Hz})$ and gamma $(35-44 \mathrm{~Hz})$. Spectral entropy is an index computed from these raw electroencephalographic signals via a series of algorithms including Fourier transformation, through which the irregularity of complicated EEG signals is converted into simplified parameters, RE and SE, in a digital form between 0 (complete regularity) and 1 (maximum irregularity) [13]. RE and SE are calculated over a frequency range between 0.8 and $47 \mathrm{~Hz}$, and between 0.8 and $32 \mathrm{~Hz}$, respectively. RE covers both EEG and EMG components, while SE includes only the EEG 
dominant area of the spectrum [13]. However, unlike the raw EEG, the spectral entropy monitoring cannot verify the activity of each brain wave because the spectral entropy values are computed by integrating total power of pertinent EEG waves into a single form by index.

The EEG power density in each brain wave frequency is changeable according to the severity $[4,6,14]$ and the type of anxiety $[5,15]$. There is a significant correlation between the raw EEG activity and the levels of anxiety in the non-anesthetized healthy patients $[6,16]$. In a study that investigated the impact of state anxiety on brain waves in a healthy group, higher state anxiety was reportedly related to an increase in delta wave power [16]. Also, trait anxiety in adult healthy subjects has been significantly correlated with the power of the beta wave on EEG although having no correlation with alpha wave activity [6]. However, higher trait anxiety in patients with social phobia brings about an increase in alpha wave activity but a decrease in theta wave power [17].

The propofol concentration at the site of use plays a role in modulating the brain wave activity of EEG in a dose-dependent manner [18-21]. Also, spectral entropy values have been positively correlated to a plasma concentration of propofol above $2.5 \mu \mathrm{g} / \mathrm{ml}$ [22] although the values of RE and SE have not been correlated with the levels of effect site concentration of propofol below $2.0 \mu \mathrm{g} / \mathrm{ml}$ [23]. However, our study revealed that the use of propofol alone at an effect site concentration between $2.0-5.1 \mu \mathrm{g} / \mathrm{ml}$ was not an influential factor on the RE and SE values (Table 5 and 6). A prior model did not exclude the effects of opioid, muscle relaxants and intraoperative stimulations on spectral entropy values at a propofol plasma concentration between 2.5 and $5 \mu \mathrm{g} / \mathrm{ml}$, which was similar to the propofol concentration of our study [22]. Thus, we presume that a reason of why the result of our study was different from the one of previous study [22] is likely the presence or absence of the effects of perioperative medications on RE and SE. Using propofol alone for anesthetic induction, based on the results of our study, we figured out that the spectral entropy values were not interfered by a propofol Ce between $2.0-5.1 \mu \mathrm{g} / \mathrm{ml}$.

Increased anxiety is associated with increased intraoperative anesthetic requirements and induction doses; especially, higher trait anxiety is significantly associated with propofol requirements in reaching a deep level of sedation and introperative anesthetic requirements under BIS monitoring [11,12]. Presently, however, no differences were found for propofol requirements at LOC between low, medium and higher anxiety groups in both state anxiety and trait anxiety under entropy monitoring. In the higher state anxiety group, because their entropy values were higher than the low and medium anxiety groups at LOC, we might give more propofol to meet the recommendation level of entropy (40-60). Thus, when determining the propofolinduced LOC by spectral entropy, anxiety levels should be considered

This study had some limitations. First, Ce at the point of LOC was designated to correspond to an OAA/S scale $<2$ and the $\mathrm{OAA} / \mathrm{S}$ scale was administered every $30 \mathrm{sec}$. Thus, in stimulating the patients, there is a possibility of more propofol requirement. Second, the initial setting of $\mathrm{Ce} 0.5 \mu \mathrm{g} / \mathrm{ml}$ with increments of 0.5 , 0.3 and $0.1 \mu \mathrm{g} / \mathrm{ml}$ as the score of the OAA/S scale reached 4,3 and 2, so the total time to LOC was too long.

Despite these limitations, our study reveals that the preoperative state anxiety plays a role in increasing the E-Entropy module-derived spectral entropy values, RE and SE, especially SE in the high state anxiety group, during the period of the anesthetic induction with propofol alone. Thus, when confirming the propofol-induced loss of consciousness by spectral entropy values, it may be desirable that pre-anesthetic anxiety levels should be considered.

\section{References}

1. Puri GD, Mathew PJ, Sethu Madhavan J, Hegde HV, Fiehn A. Bi-spectral index, entropy and predicted plasma propofol concentrations with target controlled infusions in Indian patients. J Clin Monit Comput 2011; 25: 309-14.

2. Anderson RE, Jakobsson JG. Entropy of EEG during anaesthetic induction: a comparative study with propofol or nitrous oxide as sole agent. Br J Anaesth 2004; 92: 167-70.

3. Hughes JR, John ER. Conventional and quantitative electroencephalography in psychiatry. J Neuropsychiatry Clin Neurosci 1999; 11: 190208.

4. Field T, Ironson G, Scafidi F, Nawrocki T, Goncalves A, Burman I, et al. Massage therapy reduces anxiety and enhances EEG pattern of alertness and math computations. Int J Neurosci 1996; 86: 197-205.

5. Andersen SB, Moore RA, Venables L, Corr PJ. Electrophysiological correlates of anxious rumination. Int J Psychophysiol 2009; 71: 156-69.

6. Pavlenko VB, Chernyi SV, Goubkina DG. EEG correlates of anxiety and emotional stability in adult healthy subjects. Neurophysiology 2009; 41: 400-8.

7. Caumo W, Schmidt AP, Schneider CN, Bergmann J, Iwamoto CW, Bandeira D, et al. Risk factors for preoperative anxiety in adults. Acta Anaesthesiol Scand 2001; 45: 298-307. 
8. Spielberger CD, Gorsuch RL, Lushene RE. Manual for the State-Trait Anxiety Inventory. Palo Alto, Consulting Psychologists Press. 1970.

9. Chernik DA, Gillings D, Laine H, Hendler J, Silver JM, Davidson AB, et al. Validity and reliability of the Observer's Assessment of Alertness/ Sedation Scale: study with intravenous midazolam. J Clin Psychopharmacol 1990; 10: 244-51.

10. Milne SE, Troy A, Irwin MG, Kenny GN. Relationship between bispectral index, auditory evoked potential index and effect-site EC50 for propofol at two clinical end-points. Br J Anaesth 2003; 90: 127-31.

11. Maranets I, Kain ZN. Preoperative anxiety and intraoperative anesthetic requirements. Anesth Analg 1999; 89: 1346-51.

12. Kil HK, Kim WO, Chung WY, Kim GH, Seo H, Hong JY. Preoperative anxiety and pain sensitivity are independent predictors of propofol and sevoflurane requirements in general anaesthesia. Br J Anaesth 2012; 108: 119-25.

13. Viertio-Oja H, Maja V, Sarkela M, Talja P, Tenkanen N, Tolvanen-Laakso H, et al. Description of the Entropy algorithm as applied in the Datex-Ohmeda S/5 Entropy Module. Acta Anaesthesiol Scand 2004; 48: 154-61.

14. Yang CY, Chen CH, Chu H, Chen WC, Lee TY, Chen SG, et al. The effect of music therapy on hospitalized psychiatric patients' anxiety, finger temperature and electroencephalography: a randomized clinical trial. Biol Res Nurs 2012; 14: 197-206.

15. Éismont E, Aliyeva T, Lutsyuk N, Pavlenko V. EEG correlates of different types of anxiety in 14 -to 15-year-old teenagers. Neurophysiology 2008; 40: 448-56.

16. Knyazev GG. Cross-frequency coupling of brain oscillations: an impact of state anxiety. Int J Psychophysiol 2011; 80: 236-45.

17. Sachs G, Anderer P, Dantendorfer K, Saletu B. EEG mapping in patients with social phobia. Psychiatry Res 2004; 131: 237-47.

18. Fiset P, Paus T, Daloze T, Plourde G, Meuret P, Bonhomme V, et al. Brain mechanisms of propofol-induced loss of consciousness in humans: a positron emission tomographic study. J Neurosci 1999; 19: 5506-13.

19. Wang B, Bai Q, Jiao X, Wang E, White PF. Effect of sedative and hypnotic doses of propofol on the EEG activity of patients with or without a history of seizure disorders. J Neurosurg Anesthesiol 1997; 9: 335-40.

20. Mahon P, Greene BR, Greene C, Boylan GB, Shorten GD. Behaviour of spectral entropy, spectral edge frequency $90 \%$, and alpha and beta power parameters during low-dose propofol infusion. Br J Anaesth 2008; 101: 213-21.

21. McCarthy MM, Brown EN, Kopell N. Potential network mechanisms mediating electroencephalographic beta rhythm changes during propofol-induced paradoxical excitation. J Neurosci 2008; 28: 13488-504.

22. Paolo Martorano P, Falzetti G, Pelaia P. Bispectral index and spectral entropy in neuroanesthesia. J Neurosurg Anesthesiol 2006; 18: 205-10.

23. Mahon P, Kowalski RG, Fitzgerald AP, Lynch EM, Boylan GB, McNamara B, et al. Spectral entropy as a monitor of depth of propofol induced sedation. J Clin Monit Comput 2008; 22: 87-93. 